\title{
Variations in the apparent nutrient content of foods: a study of sampling error
}

\author{
By A. W. CHAN CHIM YUK* \\ Department of Biochemistry, University of Surrey, Guildford, Surrey $\mathrm{GU}_{2}{ }_{5} \mathrm{XH}$ \\ AND ERICA F. WHEELER† AND IRENE M. LEPPINGTON \\ Department of Human Nutrition, London School of Hygiene and Tropical \\ Medicine, Keppel St, London $W C_{\mathrm{I}} E_{7} H T$ \\ (Received 8 November 1974-Accepted 4 February 1975)
}

\begin{abstract}
I. Successive portions of boiled and mashed potatoes, roast pork, cooked, freeze-dried peas and ice-cream were taken in order to determine the total (sampling and experimental) error involved in the 'duplicate analysis' method of dietary survey.

2. These samples were analysed for water, nitrogen, fat, iron and energy.

3. The experimental error of the methods was also studied separately.

4. The sampling and experimental errors ranged from $0.3 \%$ for water in potatoes, to $23 \%$ for $\mathrm{Fe}$ in ice-cream. Sampling error was significantly greater than experimental error for all nutrients studied.

5. It is concluded that in reporting the results from dietary surveys, it is necessary to take account of the errors inherent even in the most precise methods.
\end{abstract}

Many statements about 'nutrition problems' are based on the results of dietary surveys; and, more precisely, on the comparison of nutrient intakes with nutrient requirements. Consequently, it is important to know something about the accuracy with which intakes can be estimated in order to assess the significance of statements such as 'the intake of protein, calories and calcium failed to meet the recommended requirements' (Maleki, I973) or 'at least one in four of the Indian population does not get enough calories, and... possibly as much as half of the population do not have enough proteins' (Quiogue, r974).

Most dietary surveys are made by weighing or otherwise estimating food intake, and then converting food weights to nutrient intakes using tables of food composition. However, methods of this kind, although commonly used because of their relative convenience, are subject to the considerable error which is introduced by the use of food tables. They are, therefore, often standardized against a 'reference' method, which is generally taken to be the most accurate possible; that is, 'duplicate analysis'. In this method all food and drink is weighed before the subject consumes it, and an exact duplicate of the food and drink is weighed from the same serving dishes or portions of food, homogenized, and taken for chemical analysis. Thus, errors due to the variation in the composition of foods are reduced to a minimum.

The principal sources of error in estimation of nutrient intake by the 'duplicate analysis' method will therefore be: $(a)$ whatever sampling error is introduced by

* Present address: Ministry of Health, Edith Cavell St, Port Louis, Mauritius.

$\uparrow$ For reprints. 
taking successive portions of food from one batch; $(b)$ errors in weighing food; (c) errors of technique in the preparation and analysis of the homogenized food samples. Given good laboratory technique, $b$ and $c$ should not be large, but $a$ is a comparatively unknown quantity. At first sight it might seem unlikely that a large error would result from successively sampling the same batch of food. Yet, if food such as stew, fruit jelly, slices of fatty meat or fruit cake are taken, uneven distribution of fat or pieces of fruit, for example, could result in considerable sampling error. Even in 'simple' foods, such as boiled or steamed vegetables, variations in water content could produce random differences in the nutrient content of successive samples.

We have collected and analysed replicate samples of five foods (roast pork, mashed and boiled potatoes, cooked, freeze-dried peas and ice-cream) in order to determine the total error of the 'duplicate analysis' method for single foods. The foods were selected for the following reasons: pork because it has a variable fat content; boiled potatoes and peas as vegetables which might be expected to be very constant in composition; mashed potatoes because it was observed that butter or margarine were frequently but randomly added just before serving; ice-cream as an example of a homogeneous manufactured food. Thus, we expected to find the greatest sampling errors in pork and mashed potatoes, and least in peas, boiled potatoes and ice-cream. At the same time, we studied the experimental error of the laboratory methods which we were using, by repeated analyses of one homogenized sample of mashed potatoes.

\section{METHODS}

\section{Collection of food samples}

All the foods were obtained from a college refectory at lunch-time. Previously weighed containers were taken to the kitchen on different occasions, and one of the staff served out ten successive portions of one of the following foods: roast pork, boiled potatoes, mashed potatoes or peas. Ten small, wrapped ice-cream blocks were taken from one batch in the freezer. In addition, ten portions of mashed potato were all put into one container and mixed together. The kitchen staff were asked to serve out the food in exactly the same way as usual, and they were not aware of any preoccupation with the homogeneity of the food.

\section{Analytical procedures}

The samples were weighed, and then homogenized using a domestic blender, with the addition of glass-distilled water if necessary. All containers were washed in acid and glass-distilled water before use. To determine water content, a weighed portion of homogenate was dried in an air oven to constant weight at $95^{\circ}$; the remainder was deep-frozen for iron analysis. The dried material was re-homogenized using a small coffee grinder, and kept for all other analyses.

Nitrogen was determined by semi-micro-Kjeldahl digestion with concentrated sulphuric acid and a sodium sulphate-selenium catalyst, and the amount of ammonium sulphate in the digest was determined using an autoanalyser (Fleck \& Munro, 1965). 
Table I. Mean values with their standard errors and coefficients of variation $(C V)$ for nutrients and energy (/kg wet homogenate) in ten replicate analyses of one sample of mashed potato

\begin{tabular}{lclc} 
& Mean & \multicolumn{1}{c}{ SE } & CV \\
Water (g) & 735 & 0.420 & 0.18 \\
Nitrogen (g) & 2.89 & 0.0175 & $\mathrm{I} \cdot 18$ \\
Fat $(\mathrm{g})$ & 68.2 & 0.259 & $\mathrm{I} \cdot 20$ \\
Iron (mg) & 7.6 & 0.201 & 8.36 \\
Energy (MJ) & 5.59 & 0.0953 & 0.54
\end{tabular}

Fat content was determined by the Soxhlet method, extracting with light petroleum (Pearson, 1962).

Fe was determined by the method of Wootton (1958) and Hegarty (1966), in which the material is digested with concentrated nitric acid, and colour is developed by the addition of perchloric acid. To minimize contamination of samples, all glassware was washed in dilute hydrochloric acid and glass-distilled water; the fume cupboard used for digestion was washed with dilute $\mathrm{HCl}$; and all metallic equipment was covered in aluminium foil.

Energy content was determined using a ballistic bomb calorimeter (Miller \& Payne, r959) with dried sucrose ( $16.5 \mathrm{~kJ} / \mathrm{g}$ ) as a standard.

\section{Statistical analysis of results}

The mean value and standard deviation was determined for each nutrient in each food, and the coefficient of variation (CV) was calculated. In order to compare the variance due to total (sampling and experimental) error with that due to experimental technique alone, the variance ratio, $F$ (greater variance estimate $\div$ lesser variance estimate), was calculated for mashed potatoes only. High values for this ratio would indicate that the variance from one source was significantly greater than that from another.

\section{RESULTS AND DISCUSSION}

Experimental error. Table $\mathrm{x}$ gives the mean values and CVs for the analyses of ten portions from one well-mixed sample of mashed potato. The experimental error of the methods used varied with the complexity of the method; water (0.2) and energy had the lowest CVs, and Fe, in which contamination of equipment and reagents is a persistent problem, had the highest CV (8). Similar values have been reported by other workers: Hawks, Bray, Voorhees, Veley, Carlson \& Dye (1940) obtained CVs of $\mathrm{I}^{-5}-3$ for $\mathrm{N}$ and water, and Miller \& Payne (1959) give CVs of $2-3$ for replicate energy measurements.

Total (sampling and experimental) error. Table 2 gives the mean values and CVs for the analyses of ten samples of each food, with the exception of $\mathrm{Fe}$ in mashed potato, which was omitted because of deterioration of the samples. The total error in estimation varied between $0.3 \%$ for water and $23 \%$ for $\mathrm{Fe}$. Generally speaking, among foodstuffs, ice-cream had the lowest error, followed by peas. Roast pork, in which successive slices of meat could not be expected to have the same fat content, was more 


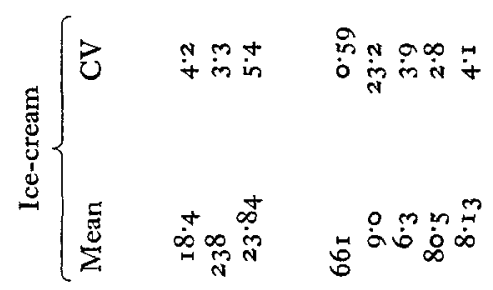

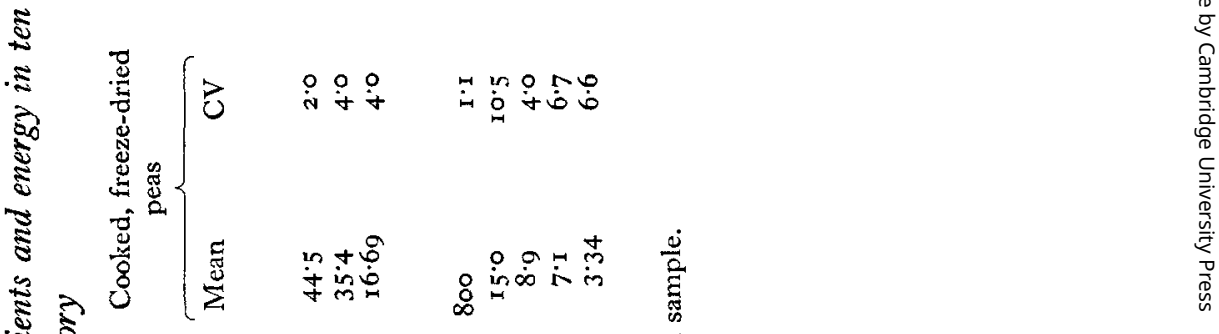

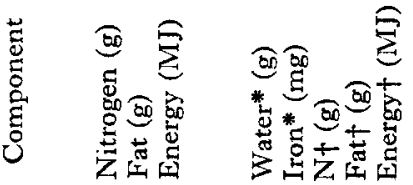


Table 3. Variance due to sampling error $\left(\mathrm{V}_{\mathrm{S}}\right)$ and experimental error $\left(\mathrm{V}_{\mathrm{E}}\right)$, and variance ratio $\left(\mathrm{V}_{\mathrm{S}}: \mathrm{V}_{\mathrm{E}}\right)$ for nutrients and energy in ten replicate analyses of one sample of mashed potato

$\begin{array}{lcccc} & & & \text { Statistical significance } \\ \text { of variance ratio: }\end{array}$

variable, as was mashed potato with added butter. The large variance for $\mathrm{N}$ in boiled potatoes was due mainly to one divergent sample and this affected the energy content also. In summary, the ranges of total (sampling and experimental) error were (\%): water $0 \cdot 3-5, \mathrm{~N}$ 2-14, fat 3-10, Fe 10-24, energy 2-6. This may be regarded as inevitable in the 'duplicate analysis' method.

Variance ratios. Table 3 gives values for the variance due to total error and the variance due to experimental error, and their ratio, for all nutrients except $\mathrm{Fe}$ in mashed potato. The values for variance ratios indicated that for all the nutrients, the variance due to total error was significantly greater than that due to experimental error. It can be assumed therefore that sampling error is the more important component of the total error.

Variation in water content. The results in Table 2, except for $\mathrm{Fe}$ and water, are expressed both per kg wet weight and per $\mathrm{kg}$ dry weight. Values per $\mathrm{kg}$ wet weight were calculated for each sample separately, using its own water content. Thus if the variance for wet food is greater than that for dry food, this can be attributed to variation in the water content of the sample. This variation, although not large, did magnify the variance of the nutrients for some foods. In the two most homogeneous foods (mashed potato and ice-cream) water content varied very little and the CVs for the nutrients were almost the same for both the dry and the wet food. For pork, boiled potatoes and peas, however, the CVs were considerably greater for the wet food than for the dry food.

These few analyses emphasize the fact that there is no way in which a subject's nutrient intake can be estimated with complete accuracy. Even if foods were homogenized thoroughly before sampling (a practical impossibility under survey conditions), some experimental error is still inevitable. Although the 'duplicate analysis' method is considered the most accurate available, and used as a reference by which others can be assessed (Marr, 1971), it is in itself subject to considerable error. The finding that water, energy, fat and $\mathrm{N}$ are estimated with more precision than $\mathrm{Fe}$ is consistent with other findings (Stock \& Wheeler, 1972).

When the results of dietary surveys are reported, it is not possible to state precisely what is the error term to be attached to the calculated or analysed results; this depends, for example, on the circumstances of the survey, the food composition tables and 


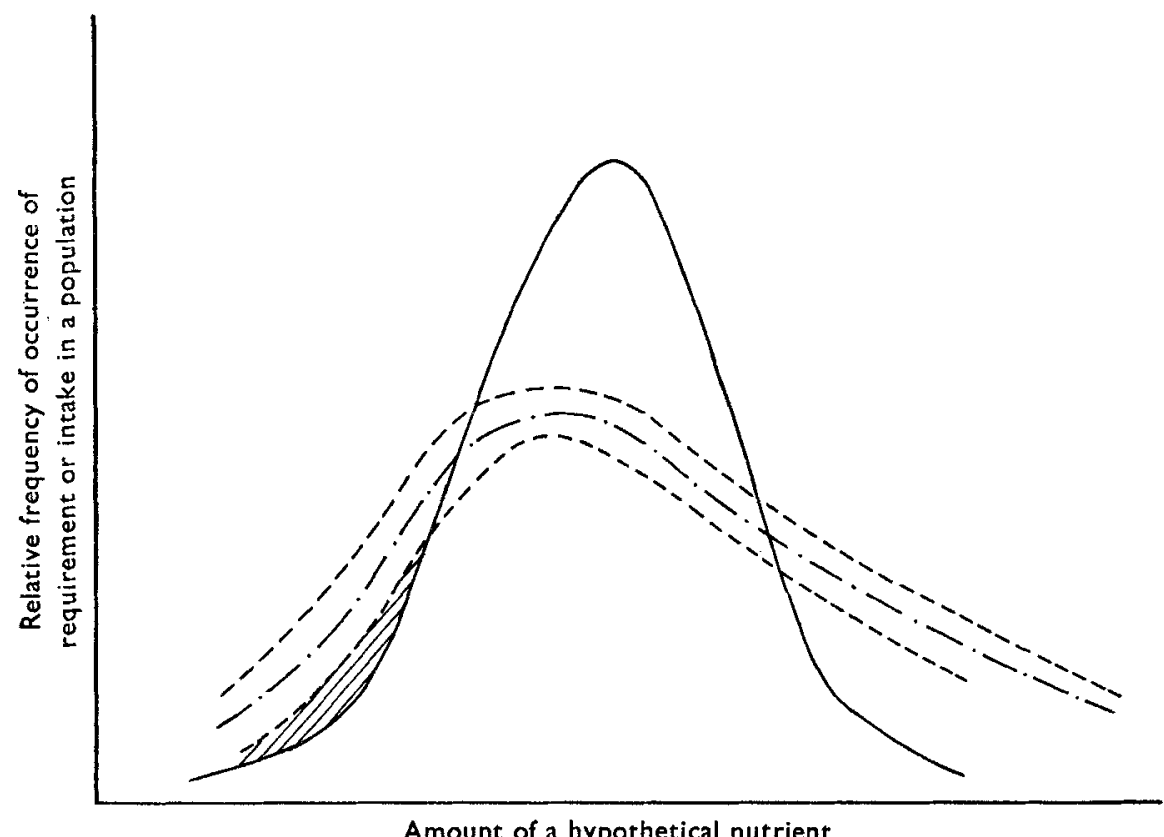

Fig. I. Distribution of the daily requirement for a hypothetical nutrient in a population $(--)$, and the distribution of the mean daily intake of the same nutrient (-...-) with limits of experimental error $(--) ; \square$, proportion of population whose daily intake is certainly less than their requirement.

analytical methods used. However, in the interpretation of these results, it is essential to bear in mind the errors of the methods involved. Fig. I presents this problem in a slightly exaggerated form. The distribution of intake and requirements are shown for any nutrient in a hypothetical population. The 'hatched' portion of Fig. $I$ is supposed to represent a proportion of the population whose intakes are certainly less than the requirement for the nutrient. Clearly the size of this 'hatched' portion of the curve depends partly on the accuracy with which intakes are estimated. Where appropriate, confidence limits should be attached to statements about numbers of individuals 'at risk' to malnutrition, or proportions of a population likely to suffer from a nutritional deficiency, if a deceptive air of accuracy is not to be attached to the conclusions drawn from dietary surveys.

\section{REFERENCES}

Fleck, A. \& Munro, H. N. (1965). Clinica chim. Acta r r, 2.

Hawks, J. E., Bray, M. M., Voorhees, J. E., Veley, B., Carlson, C. \& Dye, M. (1940). F. Am. diet. Ass. I6, 4 I6.

Hegarty, P. V. J. (r 966). Investigation of anaemogenic diets. PhD Thesis, University of London.

Maleki, M. (1973). Ecol. Fd Nutr. 2, 39.

Marr, J. W. (1971). Wld Rev. Nutr. Diet. 13, 105.

Miller, D. S. \& Payne, P. R. (1959). Br. F. Nutr. 13, 501.

Pearson, D. (1962). The Chemical Analysis of Foods, London: J. \& A. Churchill.

Quiogue, E. S. (I 974). Proc. Ist Asian Congr. Nutr., Hyderabad p. 2 r4.

Stock, A. L. \& Wheeler, E. F. (1972). Br. F. Nutr. 27, 439.

Wootton, I. D. P. (1958). Biochem. F. 68, 197. 